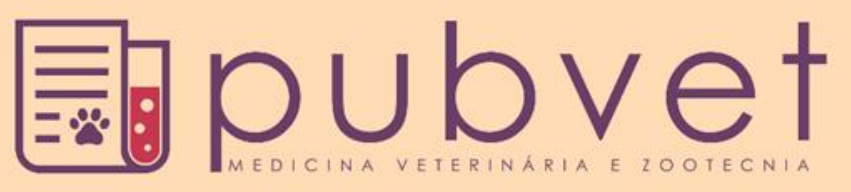

https://doi.org/10.31533/pubvet.v15n05a817.1-7

\title{
Hemangiossarcoma renal unilateral em cão: Relato de caso
}

\author{
Érika Andrade ${ }^{1 \oplus}$, Suélen Dalegrave $2^{2 *}$, Juliana Andrade ${ }^{3 \bullet}$, Laís Rezzadori Flecke ${ }^{4} \bullet$ \\ ${ }^{I}$ Médica veterinária, Caxias do Sul, RS, Brasil. \\ ${ }^{2}$ Aprimoranda do Programa de Aprimoramento de Clínica Médica e Cirúrgica de Pequenos Animais, Pontifícia Universidade Católica do Paraná, Toledo, Paraná, Brasil. \\ ${ }^{3}$ Discente em medicina veterinária, pela Universidade de Caxias do Sul, Caxias do Sul, RS, Brasil. \\ ${ }^{4}$ Aprimoranda do Programa de Aprimoramento de Clínica Médica de Pequenos Animais, Universidade de Caxias do Sul, Caxias do Sul, Rio Grande do Sul, Brasil. \\ *Autor para correspondência, E-mail: suhdalegrave@hotmail.com
}

Resumo. As neoplasias renais são incomuns e representam cerca de 0,5 a $1,7 \%$ do total de tumores em cães. Normalmente malignas, afetam cães de meia idade a idosos sendo geralmente unilaterais. Os sinais clínicos são inespecíficos, incluindo anorexia, prostração, perda de peso, letargia ou dor sublombar. Sinais mais específicos como hematúria macro ou microscópica associados à neoplasia podem ocorrer. O diagnóstico é realizado com auxílio de exames hematológicos, radiológicos e ultrassonográficos; sendo definitivo, o histopatológico. O tratamento de eleição é a nefrectomia, visto se tratar de um tumor maligno. O prognóstico ainda não está claramente definido, mas varia de reservado a mau. O Objetivo foi relatar o atendimento de um cão, fêmea tendo como diagnóstico definitivo, por histopatologia, hemangiossarcoma unilateral renal. $\mathrm{O}$ animal chegou para atendimento apresentando apatia e anorexia. Observou-se durante a avaliação clínica cifose, dor na palpação hipogástrica esquerda e região lombar. No exame ultrassonográfico observou-se um aumento de volume, anecogênico, apresentando internamente, pontos ecogênicos e estrutura amorfa irregular, ecogênica no rim esquerdo. Considerou-se processo neoplásico cavitário/abscesso/hematoma. Em íntimo contato com este aumento de volume e projetando-se caudalmente até as proximidades da bexiga, observou-se outro aumento de volume de aproximadamente $6,33 \mathrm{~cm} \times 3,7 \mathrm{~cm}$, irregular, hipoanecogênico, sugerindo neoplasia ou hematoma, envolvimento do ureter ou retroperitônio. Foi indicado o tratamento cirúrgico, sendo realizado a nefrectomia com retirada do ureter esquerdo, e encaminhado para histopatologia, tendo como diagnóstico definitivo, hemangiossarcoma. Este trabalho demonstra a importância do diagnóstico precoce, por meio de uma conduta clínica correta, exames de imagem e histopatológico, e assim um tratamento correto e eficaz, com resultado pela melhora do quadro clínico do paciente.

Palavras-chave: Cão, diagnóstico, hemangiossarcoma, rins, trato urinário

\section{Unilateral renal hemangiosarcoma in dogs: Case report}

Abstract. Neoplasms are uncommon and represent about 0.5 to $1.7 \%$ of all tumors in dogs. Usually malignant, they affect middle-aged dogs to the elderly being usually unilateral. Clinical signs are nonspecific such as anorexia, prostration, weight loss, lethargy, or sublumbar pain. More specific signs such as macro or microscopic hematuria associated with hemangiosarcoma may occur. Diagnosis is made with the aid of hematological, radiological and ultrasound examinations; histopathological definitive. The treatment of choice is nephrectomy, since it becomes of a malignant tumor. The prognosis is not yet clearly defined, but varies from reserved to bad. The objective was to report the care of a female dog with unilateral hemangiosarcoma renal histopathological diagnosis as a definitive diagnosis. The animal arrived for care presenting apathy and anorexia. During the clinical evaluation, cyphosis, pain in left hypogastric palpation and lumbar region was observed during clinical evaluation. On ultrasound examination, an anechogenic volume 
increase was observed, presenting internally, echogenic points and irregular amorphous, echogenic structure in the left kidney. A neoplastic cavity/abscess/hematoma process was considered. In close contact with this volume increase and protruding caudally to the vicinity of the bladder, another volume increase of approximately $6.33 \mathrm{~cm} \times 3.7 \mathrm{~cm}$ was observed, irregular, hypoanechogenic, suggesting neoplasia or hematoma, ureter involvement or retroperitone. Surgical treatment was indicated, with nephrectomy with removal of the left ureter referred for histopathology with hemangiosarcoma as a definitive diagnosis. This study demonstrates the importance of early diagnosis, through a correct clinical approach, imaging and histopathologic examinations and thus an effective treatment, resulting in the improvement of the patient's clinical picture.

Keywords: Dogs, diagnosis, hemangiosarcoma, kidneys, urinary tract

\section{Hemangiosarcoma renal unilateral en perros: Informe del caso}

Resumen. Las neoplasias son poco comunes y representan entre el 0,5 y el $1,7 \%$ de todos los tumores en perros. Por lo general, malignos, afectan a los perros de mediana edad a que los ancianos suelen ser unilaterales. Los signos clínicos son inespecíficos como anorexia, postración, pérdida de peso, letargo o dolor sublumbar. Pueden producirse signos más específicos como hematuria macro o microscópica asociada con hemangiosarcoma. El diagnóstico se realiza con la ayuda de exámenes hematológicos, radiológicos y ecográficos; siendo definitivo el histopatológico. El tratamiento de elección es la nefrectomía, ya que se convierte en un tumor maligno. El pronóstico aún no está claramente definido, pero varía de reservado a malo. El objetivo era reportar el cuidado de una perra con diagnóstico histopatológico renal hemangiosarcoma unilateral como diagnóstico definitivo. El animal llegó para el cuidado presentando apatía y anorexia. Durante la evaluación clínica, se observó cifosis, dolor en la palpación hipogástrica izquierda y región lumbar durante la evaluación clínica. En el examen por ultrasonido, se observó un aumento del volumen anecogénico, presentando internamente, puntos ecogénicos y estructura amorfa e irregular ecogénica en el riñón izquierdo. Se consideró un proceso neoplásico de cavidad/absceso/hematoma. En contacto cercano con este aumento de volumen y sobresaliendo caudalmente a las proximidades de la vejiga, se observó otro aumento de volumen de aproximadamente $6,33 \mathrm{~cm} \times 3,7 \mathrm{~cm}$, irregular, hipoanecogénico, sugiriendo neoplasia o hematoma, afectación del uréter o retroperitone. Se indicó un tratamiento quirúrgico, con nefrectomía con extirpación del uréter izquierdo referido para histopatología con hemangiosarcoma como diagnóstico definitivo. Este estudio demuestra la importancia del diagnóstico precoz, a través de un correcto enfoque clínico, imágenes y exámenes histopatológicos $\mathrm{y}$, por lo tanto, un enfoque terapéutico quirúrgico eficaz, lo que resulta en la mejora del cuadro clínico del paciente.

Palabras clave: Perro, diagnóstico, hemangiosarcoma, riñones, vías urinarias

\section{Introdução}

As neoplasias renais são incomuns e representam cerca de 0,5 a 1,7\% do total de tumores em cães (Fraser et al., 1996; Kahn et al., 2005). As neoplasias renais primárias têm comportamento variado e comumente são malignas (Carvalho et al., 2016), acometendo cães de meia idade a idosos (Kahn, 2013) , onde, geralmente são solitárias e unilaterais (Morris \& Dobson, 2007). Os rins, por apresentarem fluxo sanguíneo grande e uma vasta rede capilar, estão sujeitos à implantação de metástases tumorais. Por isso as neoplasias secundárias são duas vezes mais frequentes em cães do que as neoplasias renais primárias (Carvalho et al., 2016; Santos et al., 2000; Serakides \& Silva, 2016).

Hemangiossarcoma (HSA) é um tumor mesenquimal maligno com origem no endotélio dos vasos, podendo ser primário em qualquer tecido, mas raramente nos rins (Ferraz et al., 2008). Todavia, a origem renal somente é comprovada quando não há nenhum outro local com a mesma neoplasia (Serakides \& Silva, 2016). Podem apresentar diferentes tamanhos, a coloração varia de cinza-claro a vermelho-escuro, são nodulares e macios, em geral contêm áreas de hemorragia e necrose, são pobremente circunscritos, 
não encapsulados e frequentemente se aderem a estruturas adjacentes (Fernandes \& Nardi, 2016). Os HSAs geralmente crescem pela cápsula renal e pela sua fragilidade podem romper e causar considerável hemorragia (Meuten, 2016).

Os sinais clínicos são inespecíficos, como anorexia, prostração, perda de peso, letargia ou dor sublombar. Podem ocorrer sinais mais específicos, como hematúria macro ou microscópica, associados ao hemangiossarcoma. Indícios de insuficiência renal não são notados até que haja envolvimento bilateral (Morris \& Dobson, 2007; Ubukata \& Lucas, 2015).

O diagnóstico pode ocorrer com auxílio de exames hematológicos, radiológicos e ultrassonográficos (Canola et al., 2016; Kealy et al., 2012; Meuten, 2016).

A nefrectomia é indicada para tumores renais malignos, nos casos em que se apresentam unilateralmente e sem evidência de metástase. O ureter inteiro deve ser removido com o rim (MacPhail, 2014; Rosin, 1996). O prognóstico ainda não está claramente definido, mas varia de reservado a mau (Ubukata \& Lucas, 2015).

O presente estudo objetivou relatar um caso de hemangiossarcoma renal unilateral em um cão, a fim de contribuir para levantamentos de dados epidemiológicos acerca de sinais clínicos, diagnóstico e tratamento desta enfermidade.

\section{Relato de caso}

Uma cadela Pequinês de sete anos de idade, pesando seis $\mathrm{kg}$ foi atendida na clínica com a queixa de hematêmese. $\mathrm{Na}$ anamnese a tutora relatou que a paciente estava apática, anoréxica e não apresentava alterações na frequência, coloração e quantidade de urina.

Ao exame clínico constatou-se presença de cifose, dor na palpação hipogástrica esquerda e região lombar. Os demais parâmetros fisiológicos se encontravam sem alterações. Foi realizada coleta de sangue para realização de hemograma e bioquímicos tendo como resultado leucocitose, demais parâmetros dentro dos valores de referência. Para alívio rápido da dor, foi realizado a aplicação subcutânea de meloxicam $0,2 \mathrm{mg} / \mathrm{kg}$, tramadol $2 \mathrm{mg} / \mathrm{kg}$ e dipirona $25 \mathrm{mg} / \mathrm{kg}$.

No exame ultrassonográfico observou-se um aumento de volume de $4,9 \mathrm{~cm} \mathrm{x} \mathrm{4,8cm,} \mathrm{anecogênico,}$ apresentando internamente, pontos ecogênicos e estrutura amorfa irregular, ecogênica, de aproximadamente $3 \mathrm{~cm} \quad \mathrm{x} 2 \mathrm{~cm} \quad \mathrm{em}$ rim esquerdo. Considerou-se processo neoplásico cavitário/abscesso/hematoma. Em íntimo contato com este aumento de volume e projetando-se caudalmente até as proximidades da bexiga, observou-se outro aumento de volume de aproximadamente $6,3 \mathrm{~cm} \times 3,7 \mathrm{~cm}$, irregular, hipoanecogênico, sugerindo neoplasia ou hematoma, envolvimento do ureter ou retroperitônio. Foi aconselhado a realização de biopsia. Os demais órgãos não apresentavam alterações (Figura 1).

$\mathrm{Na}$ evidência de tais achados, o animal foi submetido a laparotomia exploratória. O protocolo anestésico teve como medicação pré-anestésica, morfina $(0,5 \mathrm{mg} / \mathrm{kg})$ via intramuscular. A indução anestésica foi feita com propofol $(4 \mathrm{mg} / \mathrm{kg})$, e a manutenção deu-se com isoflurano em associação à infusão contínua de fentanil $(0,03 \mu \mathrm{g} / \mathrm{kg} / \mathrm{min})$, lidocaína $(50 \mu \mathrm{g} / \mathrm{kg} / \mathrm{min})$ e cetamina $(10 \mu \mathrm{g} / \mathrm{kg} / \mathrm{min})$ usando bomba de infusão.

Na laparotomia exploratória fez-se uma incisão abdominal na linha média a partir do processo xifoide até o umbigo. Deslocando as alças intestinais para a direita e ampliando a área de visibilidade cirúrgica, com o auxílio de um afastador Farabeuf, obteve-se a visualização do rim esquerdo. O órgão estava deformado e com aumentos de volume sob a cápsula renal, formando estruturas nodulares, vermelhas e macias (Figura 2). $\mathrm{O}$ aspecto era sugestivo de neoplasia renal e optou-se pela realização da nefrectomia. Com o auxílio de uma seringa de $20 \mathrm{~mL}$ foi realizada a tentativa de drenar o conteúdo tumoral para um melhor o acesso à região do hilo renal. O resultado foi a diminuição do volume do rim e o conteúdo drenado eram coágulos e líquido sanguinolento. Após esta intervenção, a gordura perirrenal na superfície lateral dorsal do hilo foi rebatida para expor a artéria e veia renais. Estas foram separadas e ligadas individualmente com duplas ligaduras com fio de sutura Poligalactina 910 2-0, ligou-se também o ureter. Transeccionou-se as estruturas e removeu-se o rim e o ureter que foram encaminhados para avaliação histopatológica (Figura 3). 
$\mathrm{Na}$ prescrição pós-operatória instituiu-se o uso de morfina $0,5 \mathrm{mg} / \mathrm{Kg}$ intravenosa, e por via subcutânea meloxicam $0,2 \mathrm{mg} / \mathrm{kg}$ e dipirona $25 \mathrm{mg} / \mathrm{kg}$. Na internação foi adicionado como protocolo de apoio por via intravenosa ceftriaxona $30 \mathrm{mg} / \mathrm{kg}$ e metronidazol $20 \mathrm{mg} / \mathrm{kg} \mathrm{IV}$. Após dois dias internada teve alta médica.

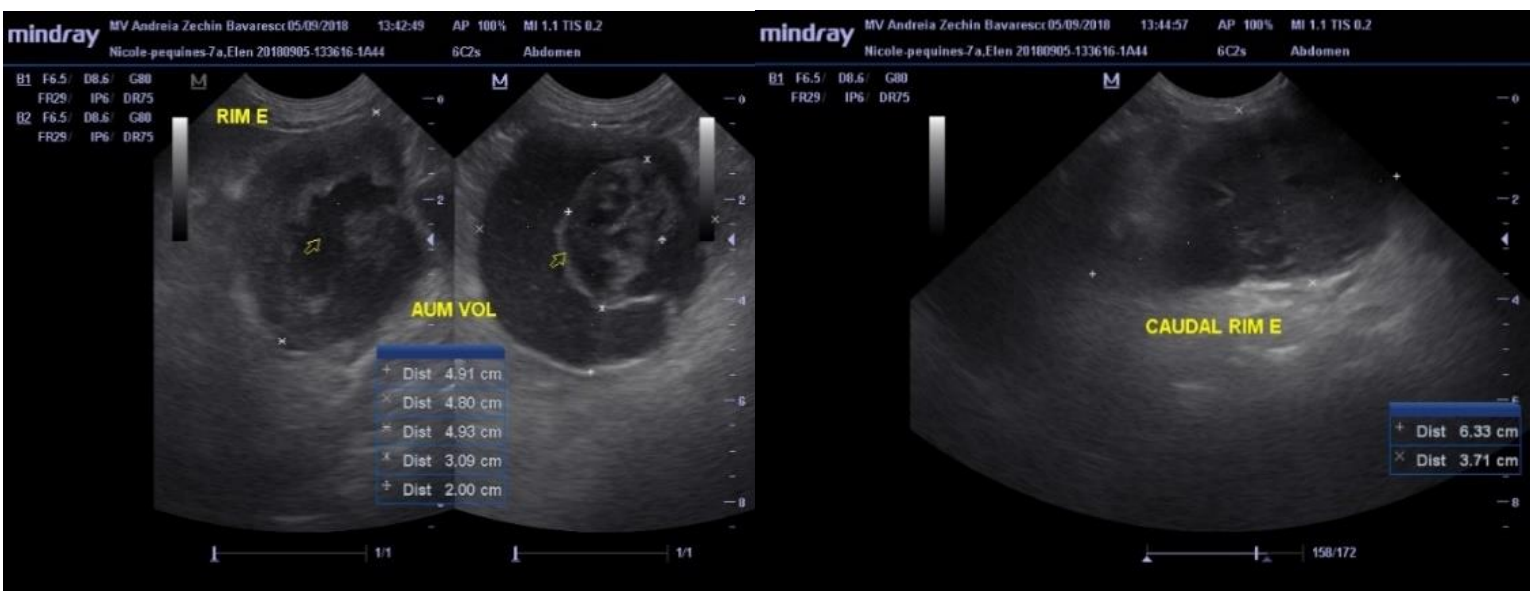

Figura 1. Ultrassonografia abdominal: (A) observou-se em rim esquerdo um aumento de volume de $4,9 \mathrm{~cm} \times 4,8 \mathrm{~cm}$, internamente outra estrutura de $3 \mathrm{~cm} \times 2 \mathrm{~cm}$. (B) - caudalmente, identificouse mais um aumento de volume de $6,33 \mathrm{~cm} \times 3,7 \mathrm{~cm}$

O diagnóstico histopatológico demonstrou proliferação neoplásica de células endoteliais imaturas, invadindo cápsula renal e obliterando o parênquima cortical. Os achados foram compatíveis com hemangiossarcoma moderadamente diferenciado. No tecido adiposo perirrenal estava presente; porém, não foi detectada invasão do tecido adiposo do seio renal, da veia renal, da pelve renal e do ureter.

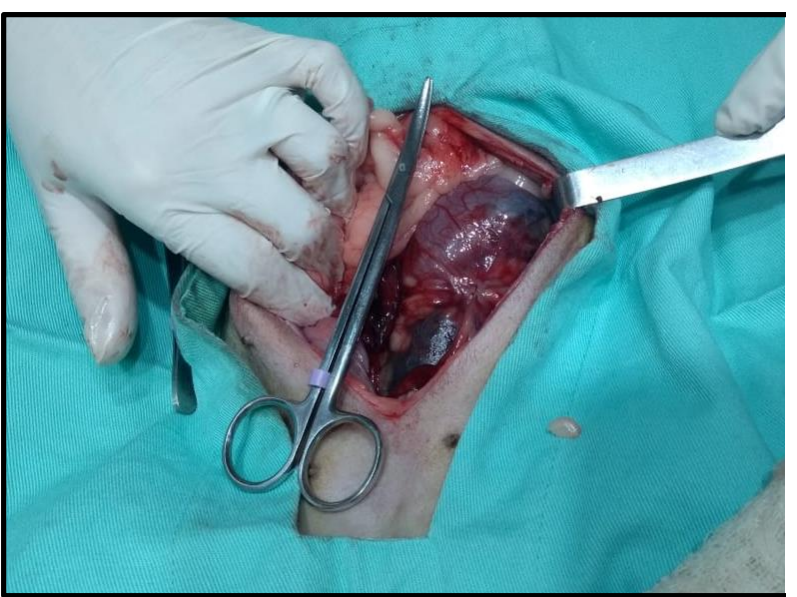

Figura 2. Canina, Pequinês, $6 \mathrm{Kg}, 7$ anos de idade. $\mathrm{Na}$ laparotomia exploratória, o rim estava deformado e com aumentos de volume sob a cápsula renal, formando estruturas nodulares, vermelhas e macias

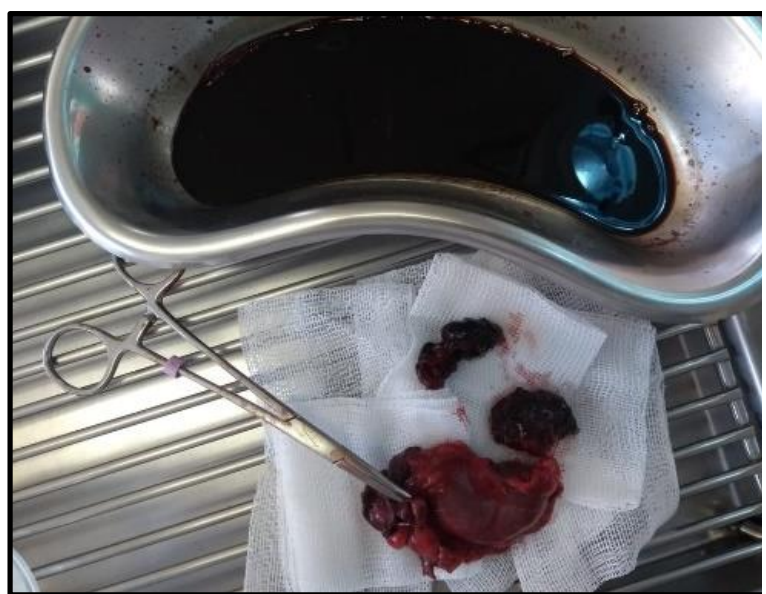

Figura 3. Canina, Pequinês, $6 \mathrm{~kg}, 7$ anos de idade. Na cuba e nas setas observa-se o conteúdo tumoral drenado durante o procedimento cirúrgico. Após este processo, realizou-se a nefrectomia unilateral do rim esquerdo e ureterectomia.

O diagnóstico histopatológico demonstrou proliferação neoplásica de células endoteliais imaturas, invadindo cápsula renal e obliterando o parênquima cortical. Os achados foram compatíveis com hemangiossarcoma moderadamente diferenciado. No tecido adiposo perirrenal estava presente; porém, não foi detectada invasão do tecido adiposo do seio renal, da veia renal, da pelve renal e do ureter.

Decorridos dez dias, foram removidos os pontos e a paciente estava se alimentando normalmente, com os parâmetros normais, não demonstrando dor. Na consulta de revisão a paciente foi encaminhada para um oncologista a fim de realizar uma avaliação e possível protocolo quimioterápico. A tutora foi alertada da importância de realizar o monitoramento da paciente. 


\section{Discussão}

Hemangiossarcomas são neoplasias malignas e correspondem a cerca de 5\% dos tumores em cães (Flores et al., 2012; Smith, 2003; Soares et al., 2017). A idade média de cães portadores desta neoplasia é de 8 a 13 anos, a paciente deste caso apresentava idade aproximada de sete anos (Smith, 2003).

$\mathrm{O}$ tumor pode iniciar em qualquer tecido vascularizado, contudo as maiores incidências primárias são no baço, átrio direito, tecido subcutâneo e fígado. Além desses locais há relatos de tumores em pulmões, pele, aorta, rins, cavidade oral, músculos, ossos, vesícula urinária, intestinos, língua, próstata, vulva, vagina, conjuntiva e peritônio (Brown et al., 1985; Ettinger et al., 2017). As formas viscerais têm maior percentual de ocorrência do que as formas não viscerais. Existem muitas divergências em relação à classificação primária ou multicêntrica do tumor (Flores et al., 2012). Em um levantamento realizado por Inkelmann et al. (2011) de neoplasmas metastáticos e multicêntricos que afetam o sistema urinário de 86 cães necropsiados demonstrou que as neoplasias metastáticas representaram um número três vezes maior que as neoplasias primárias no sistema urinário. No caso relatado, a diferenciação do HSA renal primário ou secundário não foi possível; porém, com base no histórico clínico, e na ausência de nódulos tumorais em órgãos abdominais descartam como origem tumoral o fígado, baço ou pele.

As neoplasias renais são raras e causam sinais inespecíficos, locais ou sistêmicos. A canina apresentava dor na região sublombar, corroborando com a descrição da literatura. Ela não teve azotemia, provavelmente devido ao fato de o comprometimento renal ser unilateral (Bryan et al., 2006; MacPhail, 2014). Um HSA renal pode causar hematúria (Wang \& Su, 2001), mas não foi relatado esta alteração urinária. A hematêmese pode ter como possiblidades úlceras gástricas e alterações na coagulação.

Uma avaliação minuciosa do estadiamento é essencial para planejamentos e prognósticos precisos do tratamento (Owen, 1980; Pastor, 2002). O estadiamento consiste em realizar uma série de avaliações: hemograma completo, perfil bioquímico e de coagulação, urinálise, radiografias torácicas, ecocardiograma, eletrocardiograma e ultrassonografia abdominal (Pastor, 2002). Os exames complementares realizados para a paciente foram o hemograma, perfil bioquímico e o exame ultrassonográfico abdominal. Os demais não foram realizados pela restrição financeira da tutora.

As radiografias torácicas simples e um ecocardiograma teriam sido de extrema importância para o estadiamento do quadro oncológico. Diferentes projeções torácicas podem revelar metástases pulmonares (Canola et al., 2016; Gomez et al., 2016; Smith, 2003) ou uma massa na base cardíaca indicando possível hemangiossarcoma (Fiorini et al., 2017; Smith, 2003).

O exame ultrassonográfico é um bom método de diagnóstico de massas abdominais e traz importante contribuição à determinação da origem da lesão, e na visualização de nódulos sugestivos de metástase em diferentes órgãos (Garcia et al., 2012). Em um estudo com 43 cães, a ultrassonografia indicou corretamente como neoplasia renal 84,6\% dos casos (Slatter, 2007). A realização do exame foi fundamental para o diagnóstico e para a decisão de laparotomia exploratória. As imagens do abdômen não revelaram alterações em outros órgãos abdominais, nem o aumento de volume além dos identificados no rim esquerdo.

A nefrectomia e ureterectomia parcial são recomendadas por Slatter (2007) caso o rim contralateral não apresente neoplasia e tenha função adequada. Com os indícios clínicos e os exames complementares apontando morfologia e função normal do rim direito, o tratamento cirúrgico de nefrectomia e ureterectomia unilateral esquerda foi realizado. Não foram evidenciados tumores macroscópicos nos órgãos abdominais no momento da cirurgia exploratória. Este achado coloca duas possibilidades: a possibilidade de ser um hemangiossarcoma primário renal e elimina a possibilidade de órgãos abdominais terem provocado metástase renal. Em 14 cães com HSA renal que foram submetidos a nefrectomia tiveram uma média de 278 dias de taxa de sobrevida (Locke \& Barber, 2006).

Cães com HSA renal têm maior tempo médio de sobrevida do que aqueles com formas esplênicas, cardíacas e retroperitoniais (Locke \& Barber, 2006; Meuten, 2016). Embora a quimioterapia seja geralmente recomendada, seu papel no tratamento de HSAs localizados nos rins está indefinido (Bryan et al., 2006; Smith, 2003).

Este relato de caso permite concluir que o exame ultrassonográfico é imprescindível para a detecção da alteração renal, associado ao exame histológico foram importantes para o diagnóstico de HSA renal. 
Mesmo não sendo localizados, o envolvimento de outros locais anatômicos com o tumor não pode ser descartado.

\section{Conclusão}

As neoplasias renais são raras em cães. No hemangiossarcoma renal unilateral sua etiologia, sinais clínicos, diagnóstico, tratamento e prognóstico continuam sendo um desafio para o clínico geral. Mais uma vez, a ultrassonografia abdominal direcionou o diagnóstico e guiou o tratamento cirúrgico da canina acometida. Conjuntamente com o exame histopatológico, foram os exames complementares que definiram o hemangiossarcoma renal.

A avaliação do paciente e conduta correta, se demonstrou de suma importância para um diagnóstico assertivo e precoce, com posterior tratamento cirúrgico e prognóstico favorável. A colaboração dos tutores agilidade na busca por atendimento veterinário foram também, fundamentais para o sucesso terapêutico.

\section{Referências bibliográficas}

Brown, N. O., Patnaik, A. K., \& MacEwen, E. G. (1985). Canine hemangiosarcoma: retrospective analysis of 104 cases. Journal of the American Veterinary Medical Association, 186(1), 56-58.

Bryan, J. N., Henry, C. J., Turnquist, S. E., Tyler, J. W., Liptak, J. M., Rizzo, S. A., Sfiligoi, G., Steinberg, S. J., Smith, A. N., \& Jackson, T. (2006). Primary renal neoplasia of dogs. Journal of Veterinary Internal Medicine, 20(5), 1155-1160. https://doi.org/10.1892/08916640(2006)20[1155:PRNOD]2.0.CO;2.

Canola, J. C., Medeiros, F. P., \& Canola, P. A. (2016). Radiografia convencional, ultrassonografia, tomografia e ressonância magnética. In C. R. Daleck, A. B. De Narde, \& S. Rodaski (Eds.), Oncologia em cães e gatos (pp. 133-135). Roca, Brasil.

Carvalho, M. B., Vasconcellos, A. L., \& Alves, M. A. M. K. (2016). Neoplasias do sistema urinário. In C. R. Daleck, A. B. De Narde, \& S. Rodaski (Eds.), Oncologia em cães e gatos (pp. 675-697). Roca, Brasil.

Ettinger, S. J., Feldman, E. C., \& Cote, E. (2017). Textbook of Veterinary Internal Medicine-eBook. Elsevier Health Sciences.

Fernandes, S. C., \& Nardi, A. B. N. (2016). Hemangiossarcomas. In C. R. Daleck, A. B. De Narde, \& S. Rodaski (Eds.), Oncologia em cães e gatos (pp. 776-796). Roca, Brasil.

Ferraz, J. R. S., Roza, M. R., Caetano Júnior, J., \& Costa, A. C. (2008). Hemangiossarcoma canino: revisão de literatura. Jornal Brasileiro de Ciência Animal, 1(1), 35-48.

Fiorini, E. A., Santos, T. C. B., Alves, L. C., Moreti, R., Hataka, A., Porto, C. D., \& Franco, R. P. (2017). Hemangiosarcoma at the base of the left atrium of a dog with mitral and tricuspid valve degenerationcase report. Brazilian Journal of Veterinary Research and Animal Science, 54(4), 425-429. https://doi.org/10.11606/issn.1678-4456.bjvras.2017.132471.

Flores, M. M., Panziera, W., Kommers, G. D., Irigoyen, L. F., Barros, C. S. L., \& Fighera, R. A. (2012). Aspectos epidemiológicos e anatomopatológicos do hemagiossarcoma em cães: 40 casos (19652012). Pesquisa Veterinária Brasileira, 32(12), 1319-1328.

Fraser, C. M., Bergeron, J. A., Mays, A., \& Aiello, S. A. (1996). Manual Merck de Veterinária: um manual de diagnóstico, tratamento, prevenção e controle de doenças para o veterinário. Roca.

Garcia, D. A. A., Froes, T. R., \& Guérios, S. D. (2012). Ultrassonografia abdominal pré-operatória em cães e gatos com suspeita de tumores abdominais. Ciência Rural, 42(1), 105-111. https://doi.org/10.1590/S0103-84782012000100017.

Gomez, J. R., Sanudo, M. J. M., \& Morales, J. G. (2016). Fígado. In J. R. Gomez, M. J. M. Sanudo, \& J. G. Morales (Eds.), Cirurgia na clínica de pequenos animais: Abdome cranial (pp. 199-241). MedVet.

Inkelmann, M. A., Kommers, G. D., Fighera, R. A., Irigoyen, L. F., Barros, C. S. L., Silveira, I. P., \& Trost, M. E. (2011). Neoplasmas do sistema urinário em 113 cães. Pesquisa Veterinária Brasileira, $31(11), 1102-1107$. 
Kahn, C. M., Line, S., \& Aiello, S. E. (2005). The merck veterinary manual. Merck \& Co. Whitehouse Station.

Kealy, J. K., McAllister, H., \& Graham, J. P. (2012). Radiologia e ultrassonografia do cão e do gato (Vol. 1). Elselvier Saunders.

Locke, J. E., \& Barber, L. G. (2006). Comparative aspects and clinical outcomes of canine renal hemangiosarcoma. Journal of Veterinary Internal Medicine, 20(4), 962-967. https://doi.org/10.1892/0891-6640(2006)20[962:CAACOO]2.0.CO;2.

MacPhail, C. M. (2014). Cirurgia dos sistemas reprodutivo e genital. In T. W. Fossum (Ed.), Cirurgia de pequenos animais. Elsevier.

Meuten, D. J. (2016). Tumors in domestic animals. John Wiley \& Sons.

Morris, J., \& Dobson, J. (2007). Trato urinário. In J. Morris \& J. Dobson (Eds.), Oncologia em pequenos animais (pp. 155-165). Roca, Brasil.

Owen, L. N. (1980). TNM Classification of tumours in domestic animals. World Health Organization.

Pastor, J. (2002). Canine Hemangiosarcoma. Clinical Update WSAVA 2002 Congress. Surgery, 131, $19-86$.

Rosin, E. (1996). Rim. In M. J. Bojrab (Ed.), Técnicas atuais em cirurgia de pequenos animais (pp. 346-351). Roca, Brasil.

Santos, R. L., Silva, C. M., Ribeiro, A. F. C., \& Serakides, R. (2000). Testicular tumors in dogs: frequency and age distribution. Arquivo Brasileiro de Medicina Veterinária e Zootecnia, 52(1), 25-26.

Serakides, R., \& Silva, J. F. (2016). Sistema urinário. In R. L. Santos \& A. C. Alessi (Eds.), Patologia veterinária. Roca, Brasil.

Slatter, D. H. (2007). Manual de cirurgia de pequenos animais (Vol. 2). Manole São Paulo.

Smith, A. N. (2003). Hemangiosarcoma in dogs and cats. The Veterinary Clinics of North America. Small Animal Practice, 33(3), 533-552. https://doi.org/10.1016/S0195-5616(03)00002-0.

Soares, N. P., Medeiros, A. A., Szabó, M. P. J., Guimarães, E. C., Fernandes, L. G., \& Santos, T. R. (2017). Hemangiomas e hemangiossarcomas em cães: estudo retrospectivo de 192 casos (20022014). Ciência Animal Brasileira, 18(e-30889), 1-10.

Ubukata, R., \& Lucas, R. R. (2015). Neoplasias do sistema urinário rins e bexiga. In M. M. Jerico, J. P. Andrade Neto, \& M. M. Kogika (Eds.), Tratado de medicina interna de cães e gatos (pp. 14931498). Roca, Brasil.

Wang, F.-I., \& Su, H. L. (2001). A renal hemangiosarcoma causing hematuria in a dog. Proceedings of the National Science Council, Republic of China. Part B, Life Sciences, 25(3), 187-192.

\section{Histórico do artigo:}

Recebido: 20 de novembro de 2020. Aprovado: 18 de dezembro de 2020 .

Disponível online: 22 de fevereiro de 2021.
Licenciamento: Este artigo é publicado na modalidade Acesso Aberto sob a licença Creative Commons Atribuição 4.0 (CC-BY 4.0), a qual permite uso irrestrito, distribuição, reprodução em qualquer meio, desde que $\mathrm{o}$ autor e a fonte sejam devidamente creditados. 